\title{
Ising Models on the Lobachevsky Plane
}

\author{
C. M. Series ${ }^{1}$ and Ya. G. Sinai ${ }^{2}$ \\ ${ }^{1}$ Mathematics Institute, Warwick University, Coventry, UK \\ ${ }^{2}$ Landau Institute for Theoretical Physics, Academy of Sciences of the USSR, Moscow, USSR
}

\begin{abstract}
We consider the Ising model on a lattice which is the orbit of a discrete cocompact group acting on the hyperbolic plane. For large values of the inverse temperature we construct an uncountable number of mutually singular Gibbs states.
\end{abstract}

\section{Introduction}

The goal of this paper is to study some Ising models on the Lobachevsky plane. Before discussing the motivation and the formulation of the results we shall give some necessary definitions.

Let $\mathbb{H}$ be the Lobachevsky plane and let $G$ be a finitely generated co-compact group of isometries of $\mathbb{H}$. We shall build our model on the Cayley graph $\hat{\mathscr{G}}$ of $G$. The graph $\hat{\mathscr{G}}$ is embedded in $\mathbb{H}$ in the following way. Choose a convex finite-sided

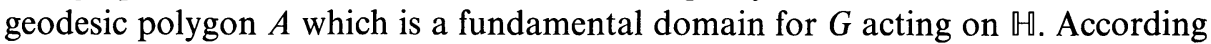
to Poincare"s theorem on fundamental polygons, the set of isometries $G_{0}$ which identify the sides of $A$ is a set of generators of $G$. Fix a point $0 \in \operatorname{Int} A$. The vertices of $\hat{\mathscr{G}}$ are the points $g 0, g \in G$. Join $g 0$ to $g^{\prime} 0$ by an edge whenever $g^{-1} g^{\prime} \in G_{0}$.

We consider spin configurations $\varphi=\{\varphi(g)\}_{g \in G}$ on $\widehat{\mathscr{G}}$, where $\varphi(g)$ takes the values \pm 1 at the vertex $g 0 \in \widehat{\mathscr{G}}$. Sites $g 0, g^{\prime} 0$ have a common bond, written $\left\langle g, g^{\prime}\right\rangle$, exactly when $g 0, g^{\prime} 0$ are joined by an edge. The Ising ferromagnetic Hamiltonian at the inverse temperature $\beta$ has the form

$$
H_{\beta}(\varphi)=-\beta \sum_{\left\langle g, g^{\prime}\right\rangle} \varphi(g) \varphi\left(g^{\prime}\right)
$$

For such models it is impossible to define the notion of free energy because for natural domains like balls the number of points belonging to the boundary is proportional to the number of points in the whole domain. However, the notion of a Gibbs state can be introduced in an unambiguous way through the DLR conditions. Namely, let $\Phi$ be the space of all configurations $\varphi=\{\varphi(g)\}_{g \in G}$, let $\mathscr{F}$ be the $\sigma$-algebra of Borel subsets of $\Phi$ and let $P$ be some probability measure defined on $\mathscr{F}$. 
Definition 1.1. $P$ is a Gibbs state corresponding to $H_{\beta}$ (see (1)) if for any finite set $V \subset G$ the conditional probability $P\{\varphi(g), g \in V \mid \bar{\varphi}(\bar{g}), \bar{g} \in G \backslash V\}$ is given by

$$
\frac{1}{\Xi(V, \bar{\varphi})} \exp \left\{\beta / 2 \sum_{\substack{\left\langle g_{1}, g_{2}\right\rangle \\\left(g_{1} \cup g_{2}\right) \cap V \neq \varnothing}} \varphi\left(g_{1}\right) \varphi\left(g_{2}\right)\right\} .
$$

Here $\bar{\varphi}=\{\bar{\varphi}(\bar{g}), \bar{g} \in G \backslash V\}$ is a fixed configuration outside $V$ and $\Xi(V, \bar{\varphi})$ is the corresponding partition function which is a normalising factor. It is clear that these conditional probabilities $P\{\varphi(g), g \in V \mid \bar{\varphi}(\bar{g}), \bar{g} \in G \backslash V\}$ depend only on those $\bar{\varphi}(\bar{g})$ for which $\bar{g} 0$ is joined by at least one edge to $V$.

Such Ising models are similar in many respects to Ising models on graphs like Cayley trees (see $[9,11]$ ). These trees may also be embedded in the Lobachevsky plane as graphs of groups of hyperbolic isometries. In the case of the modular group, similar models were constructed in [6]. (We shall explain below why we restrict our attention here to cocompact groups, for which the graph is never a tree.)

From the point of view of traditional statistical mechanics the Lobachevsky plane should be considered as an infinite dimensional space. Indeed, in the usual situations one can often write the partition function in the form

$$
\Xi(V, \bar{\varphi})=\exp \{a(\beta)|V|+\sigma(\beta)|\partial V|+r(\beta, V)\},
$$

where $a(\beta)$ is proportional to the Gibbs free energy, $\sigma(\beta)$ is associated with the surface tension and $r(\beta, V)$ is a remainder term of smaller order. For lattices in $\mathbb{R}^{d}$ the size of the surface $|\partial V|$ is of the order of $|V|^{1-1 / d}$. We use (3) as a natural definition of the thermodynamic dimension in more general situations. For example, if we consider models on $\mathbb{Z}^{d}$ with sufficiently slowly decaying interactions then this dimension can depend on the interaction and be greater than $d$. As already mentioned, for our models $|\partial V| \approx|V|$, which means that it is natural to take $d=\infty$.

The main result of this paper shows that there is another reason to believe that the Lobachevsky plane is thermodynamically infinite dimensional. Namely we prove the following result:

Theorem 1. There exists $\beta_{0}>0$ such that for all $\beta>\beta_{0}$ there exist uncountably many mutually singular Gibbs states.

The Gibbs states we construct are similar to the non-translationally invariant extremal Gibbs states for the usual Ising models on $\mathbb{Z}^{d}, d \geqq 3$, constructed by Dobrushin in [3]. His construction gives a countable number of Gibbs states and it is likely that for $d=3$ there are no others. For other $d$ it seems that there might be other Gibbs states, but apparently the total number of extremal Gibbs states is countable. Presumably the Gibbs states which we construct are extremal. Thus in our opinion, Theorem 1 also indicates the infinite dimensionality of $\mathbb{H}$ from the point of view of statistical mechanics.

Now we shall explain briefly the idea the proof of Theorem 1. Imagine the Lobachevsky plane as the unit disk with geodesics which are circular arcs orthogonal to its boundary. Fix one of these geodesics $\gamma$ and consider the sequence of domains $V_{n}=\bigcup\{g A|g \in G| g \mid, \leqq n\}$, where $|g|$ denotes the length of $g$ in the word metric on $G$ relative to the generators $G_{0}$, or equivalently the smallest number of edges in a path from 0 to $g 0$ in $\hat{\mathscr{G}}$. For all sufficiently large $n$, the domain $V_{n}$ will 


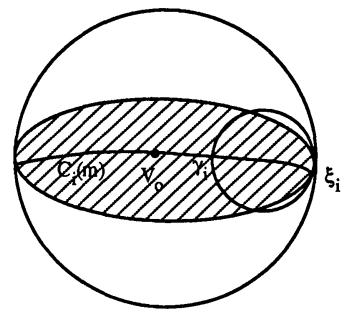

Fig. 1a

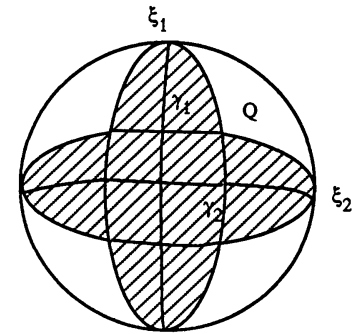

Fig. $1 b$

contain inside itself a geodesic segment $\gamma_{n}=\gamma \cap V_{n}$. Consider now the boundary condition $\bar{\varphi}_{n}=\{\bar{\varphi}(g)\}$ outside $V_{n}$, where $\bar{\varphi}(g)=+1$ if $g A$ lies on one side of $\gamma$ and $\bar{\varphi}(g)=-1$ otherwise. (We shall make this more precise in Sect. 4 below.) Using this boundary condition we can introduce the conditional Gibbs probability measure $P_{\gamma}^{(n)}$ (see (2)) on configurations $\{\varphi(g)|| g \mid \leqq n\}$.

The family $\left\{P_{\gamma}^{(n)}\right\}_{n \in \mathbb{N}}$ is compact, and has therefore at least one weak limit point which we shall denote $P_{\gamma}$. The usual arguments show that $P_{\gamma}$ is a Gibbs state. We claim that such limit points are mutually singular for different $\gamma$.

The reason for this is connected with the instability and exponential divergence of distinct geodesics in at least one direction. Take two such geodesics $\gamma_{1}, \gamma_{2}$ with a pair of distinct endpoints $\xi_{1}, \xi_{2}$ at infinity. Denote the corresponding probability measures by $P_{1}, P_{2}$. We shall show that for any $\varepsilon>0$ one can find two disjoint subsets $\Psi_{1}, \Psi_{2} \subset \Phi$ such that $P_{i}\left(\Psi_{i}\right)>1-\varepsilon, i=1,2$. This certainly implies that $P_{1}$ and $P_{2}$ are mutually singular. The construction of the sets $\bar{\Psi}_{i}$ is as follows.

Firstly, for each $m$ we define neighbourhoods $C_{i}(m)$ of $\gamma_{i}, i=1,2$. With respect to some fixed base point $v_{i}^{0} \in \gamma_{i}$ let $C_{i}(m)=\bigcup\left\{x \in \mathbb{H}: d\left(x, \gamma_{i}\right) \leqq \max \left(d\left(\pi_{\gamma_{i}}(x), v_{i}^{0}\right), m\right)\right\}$, where $\pi_{\gamma_{i}}(x)$ denotes the foot of the perpendicular from $x$ to $\gamma_{i}$, and $d$ is hyperbolic distance. (As we shall explain and carry out in detail in Sect. 4, this construction should really be done on $\mathscr{G}$ relative to the word metric on $\mathscr{G}$.) The regions $C_{i}(m)$ are illustrated in Fig. 1a.

An easy calculation shows that in a neighbourhood of $\xi_{i}, C_{i}(m)$ is contained in the horocycle based at $\xi_{i}$ through the point at distance $m$ from $v_{i}^{0}$. Thus if $\xi_{1} \neq \xi_{2}$, there is an infinite quadrant $Q$ lying outside $C_{1}(m) \cup C_{2}(m)$ and meeting $\partial \mathbb{H}$ along the entire arc from $\xi_{1}$ to $\xi_{2}$, as illustrated in Fig. $1 \mathrm{~b}$.

Now return to the conditional Gibbs distributions $P_{\gamma_{i}}^{(n)}$. In view of our boundary conditions each configuration $\varphi_{\gamma_{i}}\left(V_{n}\right)$ will have a separating contour $\Gamma_{\text {sep }}^{i}\left(\varphi\left(V_{n}\right)\right)$ which coincides with $\gamma_{i}$ outside $V_{n}$ and which connects the parts of the boundary where we have change of sign from +1 to -1 (see Fig. 2 and see Sect. 4 for details). We shall show that for all sufficiently large $n$ and $\beta$, the probability $P_{i}^{(n)}\left\{\Gamma_{\text {sep }}^{i}\right.$ lies inside $\left.C_{i}(m)\right\}>1-e^{- \text {const. } \beta m}$, where the constant depends only on $G$. This means that typical configurations $\varphi$ on one side of $C_{i}(m)$ are in the (+)-phase and on the other side are in the (-)-phase. Thus by suitable choice of boundary conditions we can arrange that in the region $Q$ described above we have, with large probability, different phases with respect to $P_{1}$ and $P_{2}$, say (+) with respect to $P_{1}$ and $(-)$ 
with respect to $P_{2}$. Now define $Q_{n}=Q \cap V_{n}$ and let

$$
\Psi_{1}=\left\{\varphi: \sum_{g \in Q_{n}} \varphi(g) \geqq \frac{3}{4}\left|Q_{n}\right|\right\}, \quad \Psi_{2}=\left\{\varphi: \sum_{g \in Q_{n}} \varphi(g) \leqq-\frac{3}{4}\left|Q_{n}\right|\right\},
$$

where $\left|Q_{n}\right|$ is the number of $g \in G$ for which $g 0 \in Q_{n}$. The necessary estimates of $P_{i}\left(\Psi_{i}\right)$ are easy to derive provided that we have already shown that $\Gamma_{\text {sep }}^{i}$ lies inside $C_{i}(m)$. In fact the technique of papers $[7,8]$ still works in our situation. This is explained in Sect. 4. Thus the hardest part is to prove the above estimate for the probability that $\Gamma_{\text {sep }}^{i}$ lies inside $C_{i}(m)$, which is done in Proposition 4.2.

We can now explain why groups whose Cayley graphs are trees embedded in the Lobachevsky plane are uninteresting from our point of view. The absence of closed loops in such a graph means that the separating contour described above is completely rigid and necessarily coincides with the $\mathscr{G}$-geodesic joining its two ends. By contrast, in the models we describe, the number of non-self intersecting paths between two points increases exponentially with the distance between the points as it does for standard lattices.

Here is the plan of the paper. In Sect. 2 we sketch the derivation of the main estimate we need from statistical mechanics which compares the contribution to the partition function from all configurations corresponding to one or the other of two separating contours, when the separating contours in question differ only by two fixed arcs whose union is a simple closed loop. The techniques here are similar to those used in $[7,8]$. In Sect. 3 we give the distance estimates we need on the graph $\hat{\mathscr{G}}$ and its dual $\mathscr{G}$. The most delicate of these estimates (Estimate 1 in Sect. 3) was derived using ideas based on Bowditch's presentation of Gromov's work on hyperbolic spaces [1]. We should like to thank Bowditch for making available to us an early version of his manuscript. Finally in Sect. 4 we prove Theorem 1.

Throughout the text we introduce many universal constants depending only on $G$. These constants will usually all be denoted by the same symbols $c$ or $k$, unless we especially need to distinguish them.

\section{Correlation Equations for Contours and Expansions of Partition Functions}

As in the introduction, let $\widehat{\mathscr{G}}$ denote the Cayley graph of some cocompact finitely generated group of isometries $G$ of $\mathbb{H}$, embedded in the Lobachevsky plane $\mathbb{H}$. The dual graph $\mathscr{G}$ of $\hat{\mathscr{G}}$ is the graph whose vertices $V(\mathscr{G})$ and edges $\Sigma(\mathscr{G})$ are the vertices and edges of the regions $g A, g \in G$. Given a configuration $\varphi$ on $\hat{\mathscr{G}}$, by a contour of $\varphi$ we essentially mean a path in the dual graph $\mathscr{G}$ whose edges are edges dual to bonds in $\hat{\mathscr{G}}$ which join points $g 0, g^{\prime} 0$ for which $\varphi$ has opposite signs. In order to ensure that contours are not self-interesting curves, and that they are uniquely defined, we in fact proceed as follows. Remove from the fundamental region $A$ small neighbourhoods of each vertex, chosen sufficiently small so as to be mutually disjoint and so as to intersect only those sides of $\mathscr{G}$ which meet at the given vertex. Denote the resulting region by $\widetilde{A}$. Transfer these neighbourhoods by the $G$ action to each vertex in $V(\mathscr{G})$. For a given configuration $\varphi$, specified outside 
the region $V_{n}$, consider the connected components of the regions

$$
\mathcal{O}_{\varphi}^{ \pm}=\bigcup\left\{g \tilde{A}: g A \in V_{n}, \varphi(g)= \pm 1\right\} .
$$

A contour of $\varphi$ is a closed non-self intersecting loop forming a connected component of the boundary of $\mathcal{O}_{\varphi}^{+}$or $\mathcal{O}_{\varphi}^{-}$. Clearly any contour can be identified with a path in $\mathscr{G}$. The number of edges of $\mathscr{G}$ in a contour $C$, written $|C|$, is called the length of $C$.

Let $C^{+}$be a contour such that immediately outside $C^{+}$we have the boundary conditions +1 and immediately inside, -1 . Introduce the partition function

$$
\Xi\left(C^{+}, \beta\right)=\sum \exp \left(\beta \sum\left\{\varphi\left(g_{1}\right) \varphi\left(g_{2}\right):\left\langle g_{1}, g_{2}\right\rangle,\left(g_{1} 0 \cup g_{2} 0\right) \cap \mathcal{O}\left(C^{+}\right) \neq \varnothing\right\},\right.
$$

where $\mathcal{O}\left(C^{+}\right)$is the set of points of $G$ lying inside $C^{+}$and the sum is over all configurations in $\mathcal{O}\left(\mathrm{C}^{+}\right)$with the given boundary conditions. As in the case of the usual Ising models (see [10]) we can rewrite this expression in terms of contours:

$$
\Xi\left(C^{+}, \beta\right)=\exp \left(\beta\left|\Delta\left(C^{+}\right)\right|-\beta\left|C^{+}\right|\right)\left(\sum \operatorname { e x p } \left(-\beta \sum\left(\left|C_{j}\right|: C_{j} \in B\left(\varphi\left(\mathcal{O}\left(C^{+}\right)\right)\right)\right),\right.\right.
$$

where $\Delta\left(C^{+}\right)$is the number of nearest neighbours $g_{1}, g_{2}$ for which $\mathcal{O}\left(C^{+}\right) \cap$ $\left(g_{1} 0 \cup g_{2} 0\right) \neq \varnothing$, and $B\left(\varphi\left(\mathcal{O}\left(C^{+}\right)\right)\right.$, the boundary of the configuration $\varphi$ in the region $\mathcal{O}\left(C^{+}\right)$, is the collection of closed contours of $\varphi$ contained in $\mathcal{O}\left(C^{+}\right)$.

The most essential term is the last sum

$$
\Xi_{0}\left(C^{+}, \beta\right)=\sum_{\varphi\left(\mathcal{O}\left(C^{+}\right)\right)} \exp \left(-\beta \sum\left(\left|C_{j}\right|: C_{j} \in B\left(\varphi\left(\mathcal{O}\left(C^{+}\right)\right)\right)\right) .\right.
$$

Differentiating $\log \left(\Xi_{0}\right)$ with respect to $\beta$ gives

$$
\frac{\partial}{\partial \beta} \log \Xi_{0}\left(C^{+}, \beta\right)=-\sum|C| \pi_{1}\left(C \mid \varphi\left(\mathcal{O}\left(C^{+}\right)\right), \beta\right),
$$

where $\pi_{1}\left(C \mid \varphi\left(\mathcal{O}\left(C^{+}\right)\right), \beta\right)$ is the first correlation function in the ensemble of contours contained inside $\mathcal{O}\left(C^{+}\right)$. A general method for studying these correlation functions and correlation functions of higher order was proposed in [7 and 8]. The basis of this method lies in the so-called system of correlation equations for contours, with the help of which the correlation functions can be represented in the form of sums of rapidly decreasing terms.

The method of correlation equations carries over without any change to the Ising model considered here on the Lobachevsky plane. In fact the basic condition for its use is the exponential upper bound on the number of contours of length $n$ passing through a given point or a given edge, which in our case is clearly satisfied. In this manner we are able to compare the partition function for different regions.

In the proof of the main theorem (cf. Proposition 4.1) we shall need the following result. Let $V_{n}$ be the region introduced in Sect. 1 and let $\bar{\varphi}_{\gamma}\left(G-V_{n}\right)$ be the corresponding boundary conditions relative to a geodesic $\gamma \subseteq \mathbb{H}$. For any configuration in our ensemble, denote by $\Gamma_{\text {sep }}=\Gamma_{\text {sep }}^{(n)}(\gamma)$ the uniquely determined separating contour, that is the contour which coincides with $\gamma$ outside $V_{n}$ and which forms the boundary between the region $(+)$ and the region $(-)$ (see Fig. 2). We denote by $\Xi\left(\beta ; V_{n}, \gamma, \Gamma_{\text {sep }}\right)$ the statistical sum over configurations for which the separating contour $\Gamma_{\text {sep }}$ is fixed. Suppose now that we are given two separating contours $\Gamma_{\text {sep }}, \Gamma_{\text {sep }}^{\prime}$, for which $\Gamma_{\text {sep }}=\Gamma_{0}+\Gamma_{1}$ and $\Gamma_{\text {sep }}^{\prime}=\Gamma_{0}+\Gamma_{2}$. In other words, $\Gamma_{\text {sep }}$ and 


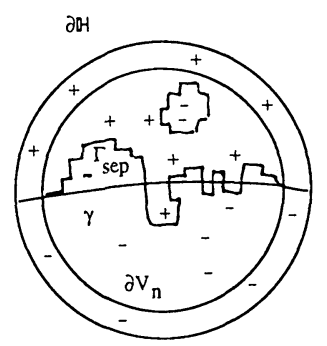

Fig. 2

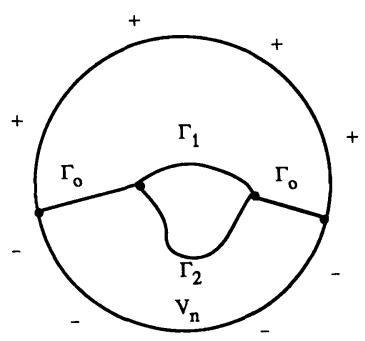

Fig. 3

$\Gamma_{\text {sep }}^{\prime}$ have a common part $\Gamma_{0}$ and differ over the (bounded) $\operatorname{arcs} \Gamma_{1}, \Gamma_{2}$. (See Fig. 3.) Then

$$
\ln \Xi_{0}\left(\beta ; V_{n}, \gamma, \Gamma_{\mathrm{sep}}\right)-\ln \Xi_{0}\left(\beta ; V_{n}, \gamma, \Gamma_{\mathrm{sep}}^{\prime}\right)=r\left(\beta ; V_{n}, \gamma, \Gamma, \Gamma^{\prime}\right),
$$

where $r$ is a remainder term which satisfies the estimate

$$
\left|r\left(\beta ; V_{n}, \gamma, \Gamma, \Gamma^{\prime}\right)\right| \leqq \text { const. } \exp \{- \text { const. } \beta\}\left(\left|\Gamma_{1}\right|+\left|\Gamma_{2}\right|\right) .
$$

This estimate follows immediately from the form of the series representing the first correlation functions for contours.

\section{Estimates from Hyperbolic Geometry}

As explained in the introduction, the main point in the proof of Theorem 1 is to show that, relative to a probability distribution $P_{\gamma}$ determined from a boundary condition associated to a geodesic $\gamma$ in $\mathbb{A}$, the separating contour lies within the region $C_{m}(\gamma)$ with probability at least $1-e^{-c \beta m}$ for $\beta$ sufficiently large and for some universal constant $c>0$. In order to make this estimate we need to measure distances in the natural graph metric on $\widehat{\mathscr{G}}$ and its dual graph $\mathscr{G}$ (described in Sect. 2). Thus for $v, v^{\prime} \in V(\mathscr{G}), d\left(v, v^{\prime}\right)$ is the minimum number of edges in a path from $v$ to $v^{\prime}$. A metric $d$ is defined on $\hat{\mathscr{G}}$ in a similar way.

It is a standard fact (see for example [2]), that both $d$ and $\hat{d}$ are pseudo-isometric to the metric $d_{\mathfrak{H}}$ induced on $\mathscr{G}, \widehat{\mathscr{G}}$ by the hyperbolic metric (denoted $d_{\sharp \mathbb{H}}$ ) on $\mathbb{H}$. In other words, there exists $k>0$, depending only on $G$, such that

$$
\frac{1}{k} d\left(v, v^{\prime}\right)<d_{\leftrightarrow}\left(v, v^{\prime}\right) \leqq k d\left(v, v^{\prime}\right) \quad v, v^{\prime} \in V(\mathscr{G}),
$$


with a similar inequality for $\hat{d}$. (The proof of this result is usually given for the Cayley graph $\hat{\mathscr{G}}$ and not for $\mathscr{G}$, however the same proof applies since there is still an upper and lower bound to the hyperbolic lengths of edges of $\mathscr{G}$, and an upper bound to the number of edges of $\mathscr{G}$ meeting at a vertex.)

We need two distance estimates, both of which are standard in the hyperbolic metric $d_{\Re}$. If $\gamma \subset \mathbb{H}$ is a geodesic and if $X \notin \gamma$, let $\pi_{\gamma}(X)$ denote the foot of the perpendicular from $X$ to $\gamma$.

Estimate $1_{\mathbb{H}}$. Let $\gamma \subseteq \mathbb{H}$ be an $\mathbb{H}$-geodesic, and let $X \notin \gamma$. Let $Y=\pi_{\gamma}(X)$ and let $Z \in \gamma$, $Z \neq Y$. Then there exists a universal constant $c$ such that

$$
d_{H}(X Z) \geqq d_{H}(X Y)+d_{H}(Y Z)-c .
$$

Estimate $2_{\boxplus}$. There exists a universal constant $J$ such that if $\gamma \subseteq \mathbb{H}$ is a geodesic and if $X, Y \notin N_{J}(\gamma)$, the $J$-neighbourhood of $\gamma$, then

$$
\rho_{J}(X, Y) \geqq \frac{3}{2} d_{\sharp}\left(\pi_{\gamma}(X), \pi_{\gamma}(Y)\right),
$$

where $\rho_{J}(X, Y)$ denotes the minimum distance from $X$ to $Y^{\prime}$ measured along paths which lie entirely outside $N_{J}(\gamma)$.

Estimate $1_{H}$ follows easily from the hyperbolic Pythagorean theorem in triangle $X Y Z$, which states that

$$
\cosh d_{H}(X Z)=\cosh d_{H}(X Y) \cosh d_{H}(Y Z) .
$$

Estimate $2_{\sharp}$ follows since perpendicular projection by a distance $u$ onto a geodesic contracts lengths by a factor sech $u$.

We shall need to use versions of Estimates 1 and 2 in which $d_{\leftrightarrow \text { }}$ is replaced by $d$ (and the term geodesic refers to a $d$-geodesic.) We need the notation of a $k$-quasi-geodesic, relative to a metric $\rho$.

Definition 3.2. A path $\gamma$ in a metric space $M, \rho$ is a $k$-quasi-geodesic if for any two points $v, v^{\prime} \in \gamma$, we have

$$
\frac{\rho\left(v, v^{\prime}\right)}{k} \leqq \rho_{\gamma}\left(v, v^{\prime}\right) \leqq k \rho\left(v, v^{\prime}\right),
$$

where $\rho_{\gamma}$ denotes distance measured along $\gamma$.

In our case either $\gamma$ is a curve in $\mathbb{H}$ and $\rho=d_{\mathbb{H}}$, or $\gamma$ is a path in $\mathscr{G}$ or $\hat{\mathscr{G}}$ and $\rho=d$ or $\hat{d}$.

Proposition 3.3. In any of the three situations above, there exists a universal constant $K=K(k)$ such that if $\lambda$ is any k-quasi-geodesic joining points $v, v^{\prime}$ then $\lambda \subset N_{K}(\gamma)$, where $\gamma$ is a geodesic from $v$ to $v^{\prime}$. This result applies even in the case when $v, v^{\prime} \in \partial \cap-$

For a proof, see for example [2]. (The proof in [2] refers to the hyperbolic metric $d_{H}$, but it is clear that the introduction of multiplicative constants using the inequality 3.1 will not affect the result.)

Notice that it follows from 3.1 that any $d$ or $\hat{d}$ geodesic is a $d_{\mathbb{H}} k$-quasi-geodesic, and thus lies at a universally bounded distance from the corresponding $\mathbb{H}$ geodesic.

The replacement of $d_{\mapsto}$ by $d$ in Estimate 2 is straightforward using 3.1 and 3.3, provided we have an analogue of the notion of the foot of the perpendicular from 
a point to a line. Given a graph geodesic $\gamma$, and a vertex $v \notin \gamma$, we can clearly construct points on $\gamma$ whose distance to $v$ is minimal. However there may be several such points, and thus to make sense of Estimate 2 we need to see that all such points are at a universally bounded distance apart. This is provided in Lemma 3.6 below. Any such point we refer to as a projection of $v$ of $\gamma$.

The situation with regard to Estimate 1 is less straightforward. The use of 3.1 introduces a multiplicative constant so that the best we can do appears to be

$$
d(X, Z) \geqq \mu(d(X, Y)+d(Y, Z))-\text { const., }
$$

where it is possible that $\mu<1$. This is unfortunately not good enough for our purposes (see the proof of Proposition 4.2). However, it is in fact true that estimate 1 remains valid relative to the metrics $d$ or $\hat{d}$, provided of course that we adjust the values of the constants suitably. This fact appears in Bowditch's presentation [1] of Gromov's work on hyperbolic spaces. For completeness we give a proof here, based on Bowditch's methods but adapted for our purposes. It will be convenient to prove the result on projections or a point to a line at the same time. We shall refer to the estimates we need relative to the graph metrices on $\mathscr{G}$ and $\widehat{\mathscr{G}}$ simply as Estimates 1 and 2.

Lemma 3.4. There exists a universal constant $c$, such that if $X Y Z$ is any geodesic triangle in $\mathbb{H}$, then there is a point $C \in \mathbb{H}$ such that $d(C, \gamma) \leqq c$, where $\gamma$ is any one of the three sides of $X Y Z$.

Proof. Let $C$ be the foot of the perpendicular from $Y$ to $X Z$. It is enough to show that there is a universal bound on $d(C,[X Y])$ and $d(C,[Y Z])$. (Here $[X Y]$ denotes the geodesic from $X$ to $Y$, etc.) This follows from the fact that there is a universal bound on the distance from the hypoteneuse of a right angled hyperbolic triangle to the opposite vertex. (This can easily be seen in the disk model by putting the right angle at the centre of the disk.)

Proposition 3.5. There exists a universal constant $c$, such that if $X Y Z$ is any geodesic triangle in $\mathscr{G}$ (or $\hat{\mathscr{G}}$ ) relative to the metric d (respectively $\hat{d})$, then there is a point $C \in \mathscr{G}$ (respectively $\widehat{\mathscr{G}}$ ) such that $d(C, \gamma) \leqq c$, where $\gamma$ is any one of the three sides of $X Y Z$.

Proof. The sides of $X Y Z$ are all $d_{\forall-1} k$-quasi-geodesics, for some universal $k>0$, and hence by Proposition 3.3 lie at a universally bounded $d_{\sharp}$-distance from the hyperbolic geodesics joining $X, Y, Z$. By Lemma 3.4, there is a point $C \in \mathbb{H}$ at universally bounded distance from these three sides. There is a vertex $C^{\prime}$ of $\mathscr{G}$ (respectively $\hat{\mathscr{G}}$ ) at bounded $d_{\mathbb{H}}$-distance from $C$, and hence at a universally bounded

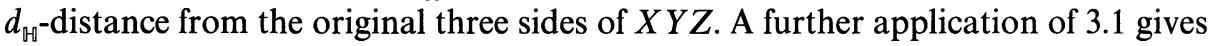
the result.

Proof of Estimate 1. Throughout the proof, we refer only to the metric $d$. Suppose that $\gamma$ is a $d$-geodesic, $X \notin \gamma$, and that $Y=\pi_{\gamma}(X)$ is some projection of $X$ on $\gamma$. Suppose also $Z \in \gamma, Z \neq Y$. Use Proposition 3.5 to find a point $C$ within bounded distance of all three sides of triangle $X Y Z$. Clearly without loss of generality we may take $C \in[X Z]$, the geodesic from $X$ to $Z$. We obtain Fig. 4, and we label the distances as shown. Using the triangle inequality in triangles $X D C$ and $Z E C$ we 


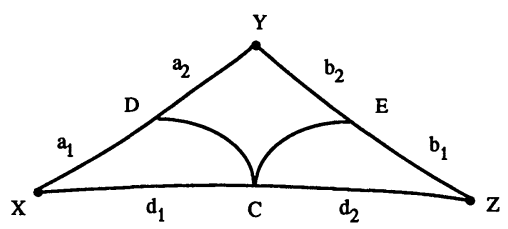

Fig. 4

see that $\left|a_{1}-d_{1}\right|$ and $\left|b_{1}-d_{2}\right|$ are universally bounded. Since $[X Y]$ is a shortest path from $X$ to $\gamma, a_{1}+a_{2} \leqq a_{1}+k+k^{\prime}$ and hence $a_{2}$ is universally bounded. It is also clear that $[X Y]$ is a shortest path from $Z$ to $[X Y]$, and hence by similar reasoning $b_{2}$ is universally bounded.

Thus $\left|a_{1}+a_{2}+b_{1}+b_{2}-\left(d_{1}+d_{2}\right)\right|$ is universally bounded, which gives the required result.

Lemma 3.6. Suppose that $\gamma$ is a geodesic in $\mathscr{G}$ (or $\widehat{\mathscr{G}})$, and that $X \notin \gamma$. Let $Y, Y^{\prime} \in \gamma$ be vertices such that $d(X, \gamma)=d(X, Y)=d\left(X, Y^{\prime}\right)$. Then there is a universal bound on $d\left(Y, Y^{\prime}\right)$, in dependent of $X$ or $\gamma$.

Proof. Let $d(X, Y)=d\left(X, Y^{\prime}\right)=a$ and let $d\left(Y, Y^{\prime}\right)=b$. By Estimate $1, a>b+a-c$, for some universal constant $c$. This immediately gives the required bound on $b$.

\section{Proof of the Main Theorem}

Let us begin by giving a precise formulation of our boundary conditions and the main results.

We claim that given any two distinct points $\xi, \eta \in \partial \Vdash$, there are both $\mathscr{G}$ and $\widehat{\mathscr{G}}$ geodesics joining them, and that any two such $\mathscr{G}$ (or $\widehat{\mathscr{G}}$ ) geodesics are at a universally bounded distance apart. (Notice that such geodesics are not necessarily unique.)

Consider the hyperbolic geodesic $\gamma$ joining $\xi$ to $\eta$. This geodesic passes through a sequence of regions..., $g_{i} A, g_{i+1} A, \ldots i \in \mathbb{Z}$. (If $\gamma$ passes through a vertex of $\mathscr{G}$ or is coincident with an edge of $\mathscr{G}$, it can obviously be replaced by a nearby curve without this property.)

Now replace $\gamma$ by the path $\tilde{\gamma}$ in the graph $\hat{\mathscr{G}}$ which joins vertices $\ldots g_{i} 0, g_{i+1} 0, \ldots$ of $\hat{\mathscr{G}}$. Using 3.1 one sees that $\tilde{\gamma}$ is a $\hat{d} k$-quasi-geodesic for some universal $k>0$. By Proposition 3.3, there is a $\hat{d}$-geodesic $\tilde{\gamma}$ within a universally bounded distance of $\tilde{\gamma}$ and hence of $\gamma$, and any two such $\hat{d}$-geodesics are at a universally bounded distance apart.

We can construct a $\mathscr{G}$-geodesic from $\xi$ to $\eta$ in a similar way, by considering a path in $\mathscr{G}$ which follows one or other side of the boundary of the chain of regions $\ldots g_{i} A, g_{i+1} A, \ldots$ cut by $\lambda$. It is clear that such a path is again a $\mathscr{G}$ quasi-geodesic,

and the same arguments as above apply.
Now let $\xi, \eta \in \partial$ H and let $\gamma$ be a $\mathscr{G}$ geodesic from $\xi$ to $\eta$. Let $V_{n}=\bigcup_{i=1}^{n} g_{i} A$ be the union of regions $g_{i} A$ such that $\hat{d}\left(g_{i} 0,0\right) \leqq n$. It is known that $V_{n}$ is a ball [4]; clearly $V_{n} \subset V_{n+1} \subset \ldots$ and $\bigcup_{n=1}^{\infty} V_{n}=\mathbb{H}$. Define $\partial V_{n}=\bigcup\left\{g_{i} A \mid d\left(g_{i} 0,0\right)=n\right\}$. Then $\gamma$ divides 


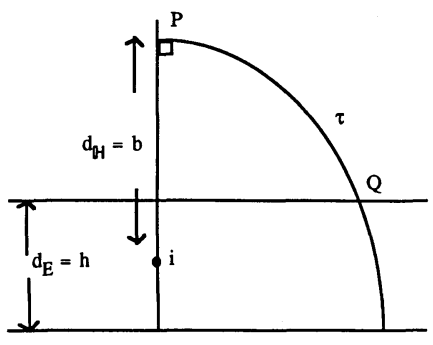

Fig. 5

$\mathbb{H}$ and $\partial V_{n}$ into two connected components which we shall for convenience refer to as "above" and "below" $\gamma$. The corresponding components of $\partial V_{n}$ are denoted by $\partial V_{n}^{+}$and $\partial V_{n}^{-}$.

The boundary condition that we use to define the probability $P_{\gamma}^{(n)}$ is that $\bar{\varphi}(g)=+1$ if $g A \subset \partial V_{n}^{+}$and $\bar{\varphi}(g)=-1$ if $g A \subset \partial V_{n}^{-}$. Alternatively, one may consider that $\bar{\varphi}(g)=+1$ if $g A \subset \mathbb{W} \backslash V_{n}$ and is above $\gamma$ and -1 otherwise.

Let now $\varphi\left(V_{n}\right)$ be any configuration on $V_{n}$, subject to the above boundary condition. Among the contours of $\varphi$, there will be one which coincides with $\gamma$ outside $V_{n}$. The part of this contour which lies inside $V_{n}$ is denoted by $\Gamma_{\text {sep }}\left(\varphi\left(V_{n}\right)\right)$, see Fig. 2. Inside the upper of the two regions separated by $\Gamma_{\text {sep }}$, the configuration is mainly in the $(+)$-phase with islands of $(-)$-phase, while below the $\Gamma_{\text {sep }}$ the situation is reversed.

We can now formulate our main results. For any $d$ geodesic $\gamma$ as above, fix a base point $v_{0} \in \gamma$. As usual, for $v \in V(\hat{\mathscr{G}}), \pi_{\gamma}(v)$ denotes a projection of $v$ on $\gamma$.

For $t>0, C_{m}(\gamma, t)=\bigcup\left\{v \in V(\mathscr{G}) \mid d(v, \gamma) \leqq \max \left(t d\left(\pi_{\gamma}(v), v_{0}\right), m\right)\right\}$. (By Lemma 3.6, the position of $\pi_{\gamma}(v)$ on $\gamma$ is defined up to a bounded universal constant, hence this definition is essentially independent of the choice of $\pi_{\gamma}(v)$.)

We claim that $t>0$ can be chosen so that there are neighbourhoods of the endpoints $\xi, \eta$ of $\gamma$ in which $C_{m}(\gamma, t)$ is contained in horocycles based at $\xi$ and $\eta$. This follows from the calculation illustrated in Fig. 5 which shows the upper half plane model with $\gamma$ as the vertical geodesic through $i$ and $\xi$ at infinity. Here $P$ is a point at hyperbolic distance $b$ above $i$, and $Q$ is the intersection of the geodesic $\tau$ perpendicular to $\gamma$ through $P$ with the horocycle at Euclidean height $h$ above the real axis. Then $d_{\mathbb{H}}(P, Q) \leqq 2 b-\log h$. We now use Proposition 3.3 and (3.1) to replace this inequality by one relative to the metric $d$, in which a multiplicative constant may have been introduced on the right-hand side. We then pick the value of $t$ so as to cancel this unwanted constant. From now on, we assume $t$ to have been chosen in this way, and write $C_{m}(\gamma)$ for $c_{m}(\gamma, t)$.

Proposition 4.1. There are universal constants $c, \beta_{0}, m_{0}$ such that

$$
P_{\gamma}^{(n)}\left\{\varphi \mid \Gamma_{\varphi}^{(n)}(\gamma) \not C_{m}(\gamma)\right\} \leqq e^{-c \beta m} \quad \text { for } \quad \beta>\beta_{0}, m>m_{0}
$$

and for all sufficiently large $n$.

The main step in proving Proposition 4.1 is the following result.

Proposition 4.2. Let $\gamma$ be a $\mathscr{G}$-geodesic, and let $v \notin \gamma$ be such that $d(v, \gamma)=h$. Let $\mu$ be $a \mathscr{G}_{-g e o d e s i c}$ from $v$ to $\pi_{\gamma}(v)$. Then there exist $\beta_{0}, c>0$ and $n_{0}$ (here $\beta_{0}, c$ are universal 
and $n_{0}$ depends on $\gamma$ ) such that

$$
P_{\gamma}^{(n)}\left\{\mu \cap \Gamma_{\varphi}^{(n)}(\gamma) \ni\{v\}\right\}<e^{-c \beta h}
$$

whenever $\beta \geqq \beta_{0}$ and $n \geqq n_{0}$.

Proof. Pick $J$ as in Estimate 2, and let $v$ denote the boundary, on the same side of $\gamma$ as $v$, of $U_{J}=\bigcup\left\{g A \mid d_{\sharp \rightarrow}(g A, \gamma) \leqq J\right\}$.

Clearly $v$ is a simple path in $\mathscr{G}$ joining the endpoints $\xi, \eta$ of $\gamma$. Suppose $n_{0}$ chosen so large that the two $\mathscr{G}_{\text {-edges }} E_{n}, F_{n}$ forming $\partial V_{n} \cap \gamma$ are at large distance from $v_{0}$ on either side of $v_{0}$ for $n \geqq n_{0}$. Let $B_{1}$ and $B_{2}$ be the nearest points on $v$ along $\Gamma_{\gamma}^{n}(\varphi)$ from $v$ in either direction from $v_{0}$, as shown in Fig. 6. (This means that $\Gamma_{\gamma}^{n}(\varphi)$ does not intersect $v$ between $B_{1}$ and $B_{2}$.) Let $\Gamma_{1}, \Gamma_{2}$ denote the arcs of $\Gamma_{\gamma}^{n}(\varphi)$ joining $B_{1}$ to $v$ and $v$ to $B_{2}$ respectively, and let $\left|\Gamma_{1}\right|,\left|\Gamma_{2}\right|$ denote the lengths of $\Gamma_{1}, \Gamma_{2}$ in the metric $d$ measured along $\Gamma_{\gamma}^{n}(\varphi)$. We need to estimate $\left|\Gamma_{i}\right|, i=1,2$. Let $B_{i}^{\prime}=\pi_{\gamma}\left(B_{i}\right), i=1,2$ denote the projections of $B_{i}$ on $\gamma$, and let $w_{0}=\mu \cap \nu$. Clearly $d\left(B_{i}, w_{0}\right)$ differs from $d\left(B_{i}^{\prime}, \pi_{\gamma}(v)\right)$ only by an additive universal constant.

By Estimate 1 we have

$$
\left|\Gamma_{i}\right| \geqq d\left(B_{i}, v\right) \geqq d\left(B_{i}^{\prime}, v\right)-c_{1} \geqq d\left(B_{i}^{\prime}, \pi(v)\right)+d(v, \pi(v))-c_{2}
$$

for some universal constant $c_{2}$.

Thus

$$
\left|\Gamma_{i}\right| \geqq d\left(B_{i}, w_{0}\right)+h-c_{3}
$$

for some universal constant $c_{3}$.

Now by construction, each $\Gamma_{i}$ lies entirely outside $N_{J}(\gamma)$. Thus by Estimate 2 we also obtain the estimate

$$
\left|\Gamma_{i}\right| \geqq \frac{3}{2} d\left(B_{i}^{\prime}, \pi(v) \geqq \frac{3}{2} d\left(B_{i}, W_{0}\right)-c_{4}\right.
$$

for a universal constant $c_{4}$.

(Notice that the estimate $\left|\Gamma_{i}\right|-d\left(B_{i}, W_{0}\right) \geqq h-$ const. of 4.2 .1 is crucial in the estimates $4.2 .4,4.2 .5$ below. It would not be strong enough simply to have a multiplicative constant multiplying the terms on the right of 4.2.1.)

We estimate the probability that $\Gamma_{\gamma}^{(n)}=\Gamma_{\gamma}^{(n)}(\varphi)$ passes through $B_{1}, B_{2}$ and $v$ by comparing the partition functions corresponding to the separating contours $\Gamma_{\gamma}^{(n)}$ and $\bar{\Gamma}_{\gamma}^{(n)}$, where $\bar{\Gamma}_{\gamma}^{(n)}$ coincides with $\Gamma_{\gamma}^{(n)}$ outside $B_{1}$ and $B_{2}$ and $\Gamma_{1} \cup \Gamma_{2}$ is replaced by the path $\Gamma_{0}=\left[B_{1} B_{1}^{\prime}\right] \cup\left[B_{1}^{\prime} B_{2}^{\prime}\right] \cup\left[B_{2}^{\prime} B_{2}\right]$, where $\left[B_{1}^{\prime} B_{2}^{\prime}\right]$ denotes the geodesic arc along $\gamma$ from $B_{1}^{\prime}$ to $B_{2}^{\prime}$ and $\left[B_{i} B_{i}^{\prime}\right]$ is a path from $B_{i} B_{i}^{\prime}$ lying inside $N_{J}(\gamma)$ and of minimal length among such paths. (It is clear from 3.1 that there is a universal upper bound to this length.)

According to (2.1), (2.2) and (2.3) in Sect. 2 we have, for contours $\Gamma_{1} \cup \Gamma_{2}$ and $\Gamma_{0}$ related as above,

$$
\frac{\Xi\left(\Gamma_{1} \cup \Gamma_{2}\right)}{\Xi\left(\Gamma_{0}\right)}=\exp \left(-c \beta\left(\left|\Gamma_{1}\right|+\left|\Gamma_{2}\right|-\left|\Gamma_{0}\right|\right)+r(\beta)\left(\left|\Gamma_{1}\right|+\left|\Gamma_{2}\right|+\left|\Gamma_{0}\right|\right)\right),
$$

where $r(\beta)<$ const. $e^{- \text {const. } \beta}$ as $\beta \rightarrow \infty$.

Here $\Xi\left(\Gamma_{1} \cup \Gamma_{2}\right)$ is the partition function for all configurations $\varphi$ whose 


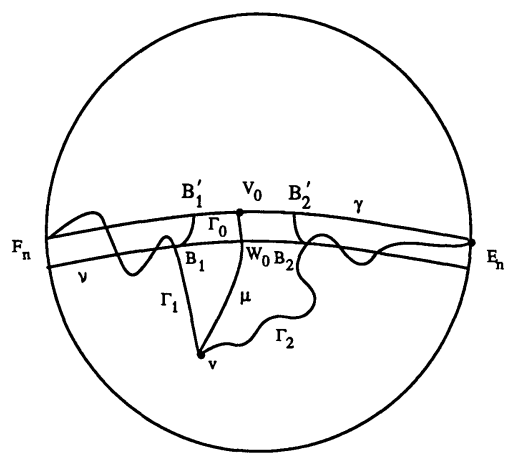

Fig. 6

separating contour contains $\Gamma_{1} \cup \Gamma_{2}$, and $\Xi\left(\Gamma_{0}\right)$ is similarly defined relative to $\Gamma_{0}$.

In the formula 4.2.3, any additive universal constant in the length estimate can be absorbed by adjusting the values of $c$ and $\beta_{0}$. Therefore we shall from now on ignore such constants in our estimates.

Choose an orientation along $\gamma$, and measure signed distance along $\gamma$ with the metric $d$ taking $v_{0}$ as the origin. We denote the co-ordinates of $B_{i}^{\prime}, i=1,2$, by $b_{i}$. Thus $\left|\Gamma_{0}\right|=\left|b_{1}-b_{2}\right|$ within a bounded universal constant.

We may then rewrite (4.2.3) in the form

$$
\frac{\Xi\left(\Gamma_{1} \cup \Gamma_{2}\right)}{\Xi\left(\Gamma_{0}\right)} \leqq \prod_{i=1}^{2}\left(-c \beta\left(\left|\Gamma_{i}\right|-\left|b_{i}\right|\right)+r(\beta)\left(\left|\Gamma_{i}\right|+\left|b_{i}\right|\right)\right) .
$$

We claim that there exists $c_{0}>0$ such that for $i=1,2$ and $\beta>\beta_{0}$ :

$$
-c \beta\left(\left|\Gamma_{i}\right|-\left|b_{i}\right|\right)+r(\beta)\left(\left|\Gamma_{i}\right|+\left|b_{i}\right|\right)<-c_{0} \beta\left|\Gamma_{i}\right| .
$$

Suppose first that $\left|\Gamma_{i}\right|<2 h$. We then use (4.2.1) to find

$$
\begin{aligned}
& (-c \beta+r(\beta))\left|\Gamma_{i}\right|+\left|b_{i}\right|(c \beta+r(\beta)) \\
& \quad \leqq-c \beta h+2 r(\beta)\left|\Gamma_{i}\right| \leqq-c^{\prime} \beta\left|\Gamma_{i}\right| \text { for suitable } c^{\prime}>0, \beta>\beta_{0} .
\end{aligned}
$$

Now say $\left|\Gamma_{i}\right|>2 h$. Then we use (4.2.2) to get

$$
\begin{aligned}
(-c \beta+r(\beta))\left|\Gamma_{i}\right|+\left|b_{i}\right|(c \beta+r(\beta)) & \leqq-c \beta\left|\Gamma_{i}\right|+c \beta\left|b_{i}\right|+2 r(\beta)\left|\Gamma_{i}\right| \\
& \leqq c_{1} \beta\left|\Gamma_{i}\right|+c \beta\left|b_{i}\right| \leqq-c_{2} \beta\left|\Gamma_{i}\right|,
\end{aligned}
$$

where we can ensure $3 c_{1}>2 c$ by choosing $\beta_{0}$ sufficiently large.

Let $\Xi\left(B_{1}, B_{2}, v\right)$ be the total contribution to the partition function from all configurations with separating contours which pass through $v$ and whose last and first intersections with $v$ on either side of $v$ are at $B_{1}, B_{2}$. Using the fact that there is an upper bound, say $q$, to the number of edges of $\mathscr{G}$ meeting at a vertex, the total number of such contours of length $r$ is bounded by $q^{r}$. Thus we obtain, for $\beta>\beta_{0}$,

$$
\frac{\Xi\left(B_{1}, B_{2}, v\right)}{\Xi\left(\Gamma_{0}\right)} \leqq \sum_{r=\left|b_{1}\right|+\left|b_{2}\right|+2 h}^{\infty} q^{r} \exp \left(-c^{\prime} \beta r\right)
$$


where we have again used the estimate (4.2.1) for $\left|\Gamma_{i}\right|$. Replacing $\beta_{0}$ if necessary by a larger value, the sum on the right is bounded above by $e^{-c^{\prime \prime} \beta h}$, for $\beta>\beta_{0}$ and some constant $c^{\prime \prime}$ independent of $\left|b_{i}\right|$.

Now the probability that we want is $\sum_{-\infty<b_{1}<b_{2}<\infty}\left(\Xi\left(B_{1}, B_{2}, v\right) / \Xi\right)$. By the above estimate, each term in this sum, and hence the whole sum, is bounded above by $e^{-c^{\prime \prime} \beta h}, \beta>\beta_{0}$, as required.

Proof of Proposition 4.1. Denote the vertices of $\mathscr{G}$ along $\gamma$ by $v_{j}, j \in \mathbb{Z}$. For each $j \in \mathbb{Z}, r \in \mathbb{N}$, let $T_{j}^{r}=\left\{v \in \mathscr{G} \mid \pi_{\gamma}(v)=v_{j}\right.$ and $\left.d\left(v, v_{j}\right)=r\right\}$. It is clear that $\left|T_{j}^{r}\right| \leqq 2 q^{r}$. (Recall that $q$ is the maximum number of edges of $\mathscr{G}$ meeting at a vertex.) Now let $\varphi$ be a configuration on $V_{n}$ with separating contour $\Gamma_{\varphi}^{(n)}$. Let $A_{j}^{r}$ be the event that $\Gamma_{\varphi}^{(n)}$ intersects $T_{j}^{r}$ but does not intersect $\bigcup_{s=0}^{r-1} T_{j}^{s}$. (Here we assume that $n \gg|j|$.)

By Proposition 4.2 and the observation above, we have

$$
P_{\gamma}^{(n)}\left(A_{j}^{r}\right) \leqq 2 q^{r} e^{-c \beta r} \leqq e^{-c_{1} \beta r} \text { for another suitable choice of } c_{1} \geqq 0, \beta_{0} .
$$

Now suppose that $\Gamma_{\gamma}^{(n)}(\varphi) \notin C_{m}(\gamma)$ (where we assume that $n \gg m$ ). Then, referring to the definition of $C_{m}(\lambda)$, the event $A_{j}^{r}$ must occur for some $j, r$ with $r \geqq \max (m,|j|)$. Thus

$$
\begin{aligned}
P_{\gamma}^{n}\left(\varphi \mid \Gamma_{\gamma}^{n}(\varphi) \notin C_{m}(\gamma)\right) & \leqq \sum_{|j|=m}^{\infty} \sum_{r=|j|}^{\infty} e^{-c_{1} \beta r}+\sum_{|j|=0}^{m-1} \sum_{r=m}^{\infty} e^{-c_{1} \beta r} \\
& \leqq c e^{-c_{1} \beta m}+2 m e^{-c_{1} \beta m}
\end{aligned}
$$

which gives the estimate we require.

Proof of Theorem 1. Now the end of the proof is simple. Take any $\varepsilon>0$. We already described in the introduction how to construct an infinite domain $Q=Q(m)$ such that

$$
\begin{array}{r}
P_{1}\left\{\Gamma_{\text {sep }}^{1} \text { lies to the left } \mathrm{Q}\right\} \geqq 1-\varepsilon, \\
P_{2}\left\{\Gamma_{\text {sep }}^{2} \text { lies below } Q\right\} \geqq 1-\varepsilon .
\end{array}
$$

(Here left, right, above, below refer to Fig. 2.) But then the usual Peierls's argument shows that $Q$ lies in the (+)-phase with $P_{1}$-probability greater than $1-\varepsilon$, i.e. $P_{1}\left\{\sum_{x \in Q} \varphi(x) \geqq \frac{3}{4}|Q|\right\} \geqq 1-\varepsilon$, and $Q$ lies in the (-)-phase with $P_{2}$-probability greater than $1-\varepsilon$, i.e. $P_{2}\left\{\sum_{x \in Q} \varphi(x) \leqq-\frac{3}{4}|Q|\right\} \geqq 1-\varepsilon$. This certainly implies the mutual singularity of $P_{1}$ and $P_{2}$.

\section{Concluding Remarks}

In the recent work of Fröhlich and Marchetti [5] some models of quantum field theory were studied in which the realisations of the field are treated as sections of a vector bundle on a Riemmanian manifold. It is a very interesting question as to how the geometry of the manifold influences the phase diagram of the model. Theorem 1 shows what kind of new possibilities may appear in this situation. 


\section{References}

1. Bowditch, B.: Notes on Gromov's hyperbolicity condition for path-metric spaces, Warwick preprint, 1989

2. Cannon, J.: The combinatorial structure of cocompact discrete hyperbolic groups. Geometriae Dedicata 16, 123-148 (1984)

3. Dobrushin, R. L.: An investigation of Gibbsian states for three-dimensional lattice systems. Teor. Veroytn. Primen. 18, 261-279 (1973)

4. Floyd, W., Plotnick, S.: Growth functions on Fuchsian groups and the Euler characteristic. Invent. Math. 88, 1-29 (1987)

5. Fröhlich, J., Marchetti, P.: Bosonization, topological solitons and fractional charges in twodimensional quantum field theory. Commun. Math. Phys. 116, 127-173 (1988)

6. Lund, F, Rasetti, M., Regge, T.: Dimer and Ising models on the Lobachevsky plane. Theor. Math. Phys. 33, 1000-1015 (1977)

7. Minlos, R. A., Sinai, Ya. G.: The phenomenon of "phase separation" at low temperature in some lattice gas models. I, Mat. Sbor. 73, 375-448 (1967) (Russ.)

8. _.: II, Trudy Mosk. Mat. Obsch. 18, 113-178 (1968) (Russ.)

9. Preston, C.: Gibbs states on countable sets. Cambridge Tracts, vol. 68, Cambridge: Cambridge University Press 1974

10. Sinai, Ya. G.: Theory of phase transitions. Rigorous results. London: Pergamon Press 1982

11. Spitzer, F.: Markov random fields on an infinite tree. Ann. Prob. 3, 387-398 (1975)

Communicated by A. Jaffe

Received April 14, 1989; in revised form July 21, 1989 